\title{
The Cancer Council Australia Medical Student Essay Competition
}

\author{
Darren Starmer $^{1}$
}

Published online: 16 August 2016

(C) American Association for Cancer Education 2016

Despite cancer being the leading cause of mortality in Australia, the depth and breadth of cancer teaching in Australian medical schools remains variable. In an effort to encourage more students to engage with oncology, the Cancer Council Australia's Oncology Education Committee [1] runs an annual essay competition.

The competition is open to all students enrolled in an Australian medical school, and the winner is sponsored to attend the World Health Organisation-Collaborating Centre for Cancer Education's International Summer School 'Oncology for Medical Students' (ISOMS). The summer school alternates between Groningen, the Netherlands and Vienna, Austria, with ISOMS 2016 being held in Groningen. The summer school runs for 2 weeks and attracts students from across the globe. The program includes tutorials, patient-led sessions, student presentations, clinical and research streams and a social program put together by local students. This journal has published papers on both the Groningen and Vienna summer schools $[2,3]$, and I encourage you to (re)visit these papers for additional information.

Each year the essay topic changes, with 'how best to teach and learn about cancer in medical schools' being chosen for 2016. In the 13 years that the competition has run, this is the first time that students have been asked to look at the issues surrounding cancer education. Previous topics have centred on more clinically focussed themes, such as multidisciplinary

Darren Starmer

darren.starmer@nd.edu.au

1 School of Medicine, The University of Notre Dame Australia, PO Box 1225, Fremantle, WA 6959, Australia cancer care, issues faced by rural and remote populations and the implications of advances in cancer research. Each topic is deliberately broad to allow students to explore the area, with the only limitation being a 2,500-word limit. Essays are judged on the concise and logical presentation of their argument, relevance to cancer care in Australia and relevance to medical students [4].

The 2016 winning essay, written by Mr. Robert McRae a final year medical student from the University of Notre Dame Australia's Sydney Medical School, has been published in its entirety in this edition of the Journal. The essay is unique in that it offers the perspective of a medical student on both the importance of cancer education and the form that such education might take. As an essay, it offers a very different insight to those which we obtain through the ubiquitous teaching evaluations or surveys. As such, there have been no editorial changes to the original essay submission, with the exception of the formatting of references.

I am sure that you will enjoy reading this essay, and for those of you who are involved in the teaching of medical students, the essay submissions that were awarded runnerup prizes are available from the Cancer Council Australia website [4].

Darren Starmer

Chair-Oncology Education Committee, Cancer Council Australia.

\section{References}

1. Cancer Council Australia. Oncology Education Committee Sydney: the Cancer Council Australia; 2016 [updated March 14, 2016; cited 2016 July 18]. Available from: http://www.cancer.org.au/healthprofessionals/oncology-education/ 
2. de Vries J, Szabo BG, Sleijfer DT (2002) The educational yield of the international summer school "Oncology for Medical Students". J Cancer Educ 17(3):115-120

3. Fromm-Haidenberger S, Pohl G, Widder J, Kren G, Fitzal F, Bartsch $R$, et al. (2010) Vienna international summer school on experimental and clinical oncology for medical students: an Austrian cancer education project. J Cancer Educ 25(1):51-54
4. Cancer Council Australia. 2016 Medical Student Essay Competition Sydney: the Cancer Council Australia; 2016 [updated June 10, 2016; cited 2016 July 18]. Available from: http://www.cancer.org.au/healthprofessionals/oncology-education/2016-medical-student-essaycompetition.html. 